\title{
Researching on real-time transmission multimedia image in Network teaching
}

\author{
Ju Hua
}

Inner Mongolia Normal University Media College,Hohhot,Inner Mongolia, 010022, China ${ }^{1}$ Inner Mongolia University School of Mongolia Studies, Hohhot, Inner Mongolia, 010022, China ${ }^{2}$

Keywords: network teaching; multimedia; image transmission;

\begin{abstract}
In the research process of real-time transmission multimedia image in Network teaching, there is a problem of poor visual effects in network teaching, due to the current algorithm for image teaching transmission has not fully consider the human visual system. A method of real-time multimedia image transmission based on improved ROI algorithm in network teaching is proposed. In this method, the network status of the teaching is judged by the feedback of the TCP data packets. ROI image transmission is carried out with the data frame length of the multimedia image transmission in the network teaching. Under the premise of not affecting the quality of the real-time transmission of ROI in the network teaching, wavelet coefficients delayed transmission is proposed with bandwidth savings to complete the real-time transmission of multimedia image. The experimental results show that the real-time transmission of multimedia image transmission method based on the improved ROI algorithm is effective, and the visual effect is good.
\end{abstract}

\section{Introduction}

With the rapid development of the Internet, people expect Internet content to be more diversified. With the development of electronic commerce, distance education and real-time monitoring technology, multimedia data transmission has played important role on the Internet business $^{[1-3]}$, people also want to introduce more multimedia data services to achieved making sound, image and text in the network teaching ${ }^{[4-6]}$. However, the amount of multimedia data is very large, the traditional control mechanism of the information transmission is difficult to meet the transmission of multimedia data ${ }^{[7]}$. Meanwhile, the technology of real-time transmission of multimedia image in network teaching is an effective way to solve this problem, which has attracted many experts and scholars' attention ${ }^{[8,9]}$. Due to the technology of network teaching in the transmission of real-time multimedia image has profound significance for development, it has become the focus of professors, has received extensive attention, at the same time, there are a lot of good method proposed ${ }^{[10]}$.

At present, the real-time transmission of multimedia image are mainly based on UDP algorithm of network, wavelet algorithm and CDMA algorithm in network teaching. Wavelet algorithm is commonly used for real-time transmission. Not fully considering the human visual characteristics, it reflects poor visual effects of network teaching.

According to the above defects, a method of real-time multimedia image transmission based on improved ROI algorithm in network teaching is proposed. In this method, the network status of the teaching is judged by the feedback of the TCP data packets. ROI image transmission is carried out with the data frame length of the multimedia image transmission in the network teaching. Under the premise of not affecting the quality of the real-time transmission of ROI in the network teaching, wavelet coefficients delayed transmission is proposed with bandwidth savings to complete the real-time transmission of multimedia image. The experimental results show that the real-time transmission of multimedia image transmission method based on the improved ROI algorithm is effective, and the visual effect is good. 


\section{Real-time transmission theory of multimedia image}

The process of real-time transmission of multimedia images in network teaching is divided into three layers: the top layer is the logic control layer as the core of the algorithm, which is responsible for the realization of the image's sub-packet and reorganization, flow control and packet loss recovery; the middle layer is a forward image transmission layer, which is used to send large amount of data from the sender to the receiver, so as to ensure the rapid transmission of data packets; the bottom layer is a backward status information transmission layer, which is used to receive feedback status information to ensure the reliable and accurate transmission of the status information.

The flow control of real-time transmission image in the network teaching is completed by the sender and receiver. The sender completes the traffic control by the data packet size DS (unit: bit), data packet sending time DT (unit: ms) and image sending timer. Specific procedures are as follows.

Provided the rectangular coordinates $\left(X_{L}, Y_{L}\right)$ and $\left(X_{R}, Y_{R}\right)$ are

$x_{L}^{\prime}=\frac{X_{L}}{2}-n e g$

$x_{R}^{\prime}=\frac{X_{R}}{2}+P O S$

$x_{L}^{\prime}=\frac{Y_{L}}{2}-n e g$

$Y_{R}^{\prime}=\frac{Y_{R}}{2}+P O S$

Based on the formula, the value of the constant neg and pos depends on two aspects, one is whether two vertices are located in low or high frequency sub-band, the other depends on original signals $\left(X_{L}, Y_{L}\right)$ and $\left(X_{R}, Y_{R}\right)$.

In summary, the real-time transmission of multimedia images in network teaching can be effectively realized.

\section{Optimization principle of real-time image transmission}

According to defects of poor visual effects in network teaching because of not fully considering the human visual system, a method of real-time multimedia image transmission based on improved ROI algorithm in network teaching is proposed.

\subsection{Characteristic extraction of frame-length data}

According TCP data package of the receiver feedback, the teaching network condition is judged. Then combined with frame length feature of ROI image transmission, image data transmission is performed. Detailed procedures are as follows.

Provided the average speed of the nth frame of image is

$$
\overline{V_{n}\left(\alpha_{n}, \beta_{n}, \mu_{n}\right)}=\sum_{o}^{n} D S_{i} / \sum_{0}^{n} D T_{i}=\frac{N C_{(n-1)} \times \alpha_{n}+D S_{(n-1)} \times\left(\mu_{n}-N F_{(n-1)}\right)}{D T_{(n-1)} \times \mu_{n} /\left(\mu_{n}-N F_{(n-1)}\right)-N C_{(n-1)} \times \beta_{n}}
$$

Where $\alpha_{n}, \beta_{n}, \mu_{n}$ are speed adjusting factors (all positive) and can be set by frame length of network data. $D S_{i}$ and $D T_{i}$ respectively means size and sending time of data packets of the ith frame. $N C_{i}$ presents transmission condition of the ith frame, which equals 1 with success transmission and 0 with failure one. 
Instantaneous velocity of the nth frame is

$V_{n}\left(\alpha_{n}, \beta_{n}, \mu_{n}\right)=D S_{n} / D T_{n}=\frac{N C_{(n-1)} \times \alpha_{n}+D S_{(n-1)} \times\left(\mu_{n}-N F_{(n-1)}\right) / \mu_{n}}{D T_{(n-1)} \times \mu_{n} /\left(\mu_{n}-N F_{(n-1)}\right)-N C_{(n-1)} \times \beta_{n}}$

The magnitude of the instantaneous speed is related to the adjustment factor, the packet loss status and the transmission speed of the previous frame. The transmitter can be adjusted according to the packet loss and the speed of the first frame to calculate instantaneous transmission rate speed. Speed adjustment factor determines the speed of image transmission in the current network teaching and the performance of the flow control.

\subsection{Realization of real-time multimedia image transmission}

So as to not affect ROI subjective quality of real-time transmission in the teaching network, ROI of wavelet coefficients could be delayed transmission with no significant effect on the visual effects. The use of transmission bandwidth savings is efficiently employed for visually teaching background coefficient to complete real-time image transmission.

Assuming wavelet inverse transform formula of $5 / 3$ integer wavelet is

$$
X(2 n)=L(n)-\frac{H(n-1)+H(N)}{4}
$$

Where $X(2 n)$ pixels in the original image.

The background transmission is carried out by the progressive ROT, and the background image is controlled by the so-called expansion factor $S(S \geq 0)$ and hierarchically transmitted in certain radius.

$\left(X_{L}, Y_{L}\right)$ and $\left(X_{R}, Y_{R}\right)$ represent right side's coordinates of rectangular ROT. $a=\min \left(\left|x_{L}-x_{R}\right|,\left|y_{L}-y_{R}\right|\right)$ and $b=\left(\left|x_{L}-x_{R}\right|,\left|y_{L}-y_{R}\right|\right)$ means length and width of rectangular or short and long axis of ellipse. Extended factor $S$ is expressed as $S=2 \Delta r$ / $a$, where $\Delta r$ is the radius of the first level background.

From the above formula, when $\mathrm{S}$ gets maximum value $S_{\max }$, i.e., the whole background is send as real-time transmission image. The value of $S_{\max }$ is not only depend on ROT but also the location of ROT in the image. As background image is extended, if any side of original image reaches the boundary, it is said that last level of extension is achieved. The rest of previous extension is obtained as the last extension to be transmitted as real-image in network teaching. $S_{\max }$ is formulated as:

$$
S_{\max }=\frac{2 \min \left(X_{L}, Y_{L}, M-X_{R}-1, N-Y_{R}-1\right)}{a}
$$




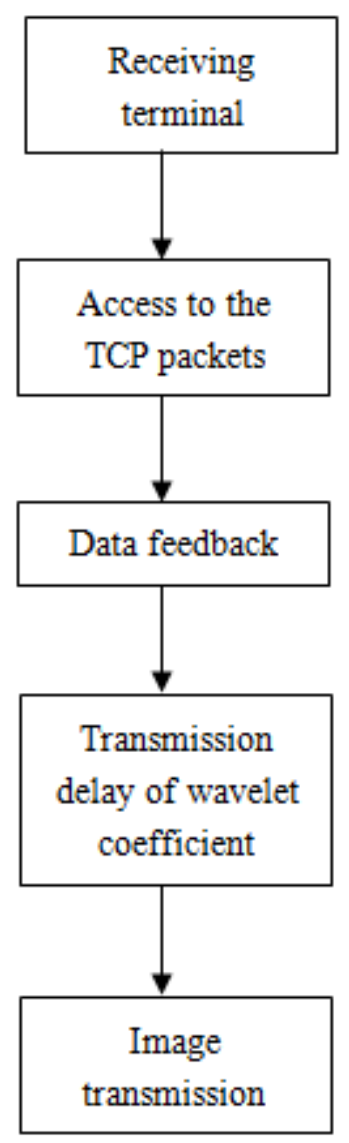

Figure 1 structure chart of multimedia image real-time transmission

In summary, the optimization principle of image real-time transmission in the network teaching multimedia is performed effectively.

\section{Experiments and simulation}

In order to prove the effectiveness of improved ROI algorithm in network teaching of multimedia real time image transmission, experiments are required. Taking standard image Lend $(256 \times 256 \times 8)$

as example and building virtual experiment environment of real-time multimedia image transmission in the MATLAB environment, experiments are performed using the modified algorithm and the traditional algorithm for real-time multimedia image transmission. The two transmission algorithms of image visual effect are compared to measure the transmission validity.

Comparative results are shown in Figure 2 and Figure 3.

\section{the first laptop}

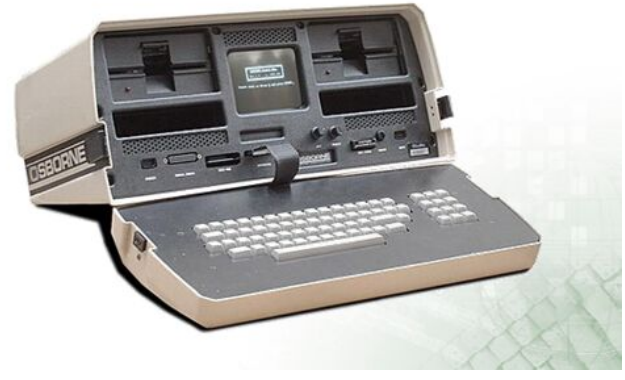

Figure 2 Image quality of using traditional algorithm for transmission 


\section{the first laptop}

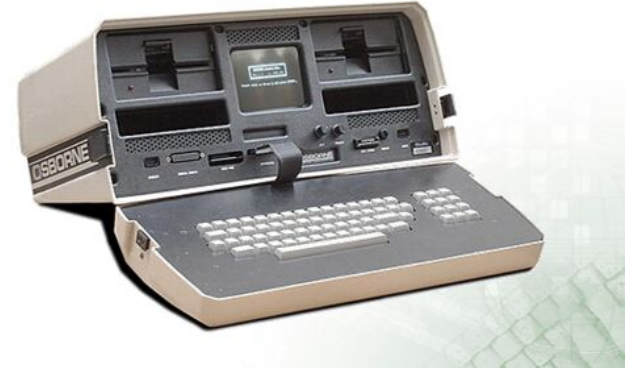

Figure 3 Image quality of using improved algorithm for transmission

In Figure 2 and Figure 3 it can be seen that the improved algorithm for real-time multimedia image transmission is obviously superior to the traditional algorithms. The experiments based on the ROI algorithm of network teaching in multimedia real-time transmission is high efficiency and its visual effect is better.

\section{5 conclusions}

According to defects of poor visual effects in network teaching because of not fully considering the human visual system, a method of real-time multimedia image transmission based on improved ROI algorithm in network teaching is proposed. In this method, the network status of the teaching is judged by the feedback of the TCP data packets. ROI image transmission is carried out with the data frame length of the multimedia image transmission in the network teaching. Under the premise of not affecting the quality of the real-time transmission of ROI in the network teaching, wavelet coefficients delayed transmission is proposed with bandwidth savings to complete the real-time transmission of multimedia image. The experimental results show that the real-time transmission of multimedia image transmission method based on the improved ROI algorithm is effective and the visual effect is good.

[基金项目] 内蒙古自治区高等学校科学技术研究项目 “利用蒙古文语料库制作蒙古文课程资源的实践研究” 的资助。（编号: NJZY14038）”的阶段性成果之一。

\section{References}

[1]Cui Yan. Research and Implementation of Image Transmission in Multimedia Network-Teaching System [J]. Journal of Luoyang normal University, 2013, 32(2):82-85.

[2]Wang Zhiyu. Advantage of multimedia and online teaching to accelerate teaching reform of junior high school chemistry [J]. Questions and research: New curriculum forum, 2014, (1):31-31.

[3]Zhu Minghui. Application and research on multimedia technology in Physical Education [J]. Charmin China, 2014, (7):223-223.

[4]Qiu Zengguo. Discussion on the video surveillance and image display system construction based on wireless transmission [J]. Satellite TV \& IP Multimedia, 2014, (1):66-67.

[5]Chen Hu. Thinking of Multimedia Teaching Application [J]. Supervised: Scholars spiritual home, 2013, (22):111-111

[6]Qin Lihua Chen Xiaoyang Chen Yan. Considerations on Multimedia Technology in classroom teaching application of Health assessment [J]. Hunan Journal of Traditional Chinese Medicine, 2013, (9):111-112.

[7]Gu Kaiji. Discussion on Misunderstandings of Multimedia Technology in the teaching process

[J]. China educational technique and equipment, 2013, (34):87-88.

[8]Yu Huazhong. Reform of multimedia network technology and teaching model under the New Curriculum [J]. Inspiration and Wisdom: Education Edition, 2013, (10):6-6.

[9] Sun Yongbing. Status and Trends of Network Multimedia Teaching [J]. 2014, 31(4):326-329. China educational technique and equipment, 2013, (8):54-55.

[10] Chen Hongxing. Realization of Network Multimedia Access with Wide Distribution [J]. Computer Simulation, 2014, 31(4):326-329. 